\title{
The Vector-Thread Architecture
}

\author{
Ronny Krashinsky, Christopher Batten, Mark Hampton, Steve Gerding, \\ Brian Pharris, Jared Casper, and Krste Asanović \\ MIT Computer Science and Artificial Intelligence Laboratory, 32 Vassar Street, Cambridge, MA 02139 \\ \{ronny, cbatten, krste\}@csail.mit.edu
}

\begin{abstract}
The vector-thread (VT) architectural paradigm unifies the vector and multithreaded compute models. The VT abstraction provides the programmer with a control processor and a vector of virtual processors (VPs). The control processor can use vector-fetch commands to broadcast instructions to all the VPs or each VP can use thread-fetches to direct its own control flow. A seamless intermixing of the vector and threaded control mechanisms allows a VT architecture to flexibly and compactly encode application parallelism and locality, and a VT machine exploits these to improve performance and efficiency. We present SCALE, an instantiation of the VT architecture designed for low-power and high-performance embedded systems. We evaluate the SCALE prototype design using detailed simulation of a broad range of embedded applications and show that its performance is competitive with larger and more complex processors.
\end{abstract}

\section{Introduction}

Parallelism and locality are the key application characteristics exploited by computer architects to make productive use of increasing transistor counts while coping with wire delay and power dissipation. Conventional sequential ISAs provide minimal support for encoding parallelism or locality, so high-performance implementations are forced to devote considerable area and power to on-chip structures that extract parallelism or that support arbitrary global communication. The large area and power overheads are justified by the demand for even small improvements in performance on legacy codes for popular ISAs. Many important applications have abundant parallelism, however, with dependencies and communication patterns that can be statically determined. ISAs that expose more parallelism reduce the need for area and power intensive structures to extract dependencies dynamically. Similarly, ISAs that allow locality to be expressed reduce the need for longrange communication and complex interconnect. The challenge is to develop an efficient encoding of an application's parallel dependency graph and to reduce the area and power consumption of the microarchitecture that will execute this dependency graph.

In this paper, we unify the vector and multithreaded execution models with the vector-thread (VT) architectural paradigm. VT allows large amounts of structured parallelism to be compactly encoded in a form that allows a simple microarchitecture to attain high performance at low power by avoiding complex control and datapath structures and by reducing activity on long wires. The VT programmer's model extends a conventional scalar control processor with an array of slave virtual processors (VPs). VPs execute strings of RISC-like instructions packaged into atomic instruction blocks (AIBs). To execute data-parallel code, the control processor broadcasts AIBs to all the slave VPs. To execute thread- parallel code, each VP directs its own control flow by fetching its own AIBs. Implementations of the VT architecture can also exploit instruction-level parallelism within AIBs.

In this way, the VT architecture supports a modeless intermingling of all forms of application parallelism. This flexibility provides new ways to parallelize codes that are difficult to vectorize or that incur excessive synchronization costs when threaded. Instruction locality is improved by allowing common code to be factored out and executed only once on the control processor, and by executing the same AIB multiple times on each VP in turn. Data locality is improved as most operand communication is isolated to within an individual VP.

We are developing a prototype processor, SCALE, which is an instantiation of the vector-thread architecture designed for low-power and high-performance embedded systems. As transistors have become cheaper and faster, embedded applications have evolved from simple control functions to cellphones that run multitasking networked operating systems with realtime video, three-dimensional graphics, and dynamic compilation of garbagecollected languages. Many other embedded applications require sophisticated high-performance information processing, including streaming media devices, network routers, and wireless base stations. In this paper, we show how benchmarks taken from these embedded domains can be mapped efficiently to the SCALE vectorthread architecture. In many cases, the codes exploit multiple types of parallelism simultaneously for greater efficiency.

The paper is structured as follows. Section 2 introduces the vector-thread architectural paradigm. Section 3 then describes the SCALE processor which contains many features that extend the basic VT architecture. Section 4 presents an evaluation of the SCALE processor using a range of embedded benchmarks and describes how SCALE efficiently executes various types of code. Finally, Section 5 reviews related work and Section 6 concludes.

\section{The VT Architectural Paradigm}

An architectural paradigm consists of the programmer's model for a class of machines plus the expected structure of implementations of these machines. This section first describes the abstraction a VT architecture provides to a programmer, then gives an overview of the physical model for a VT machine.

\subsection{VT Abstract Model}

The vector-thread architecture is a hybrid of the vector and multithreaded models. A conventional control processor interacts with a virtual processor vector (VPV), as shown in Figure 1. The programming model consists of two interacting instruction sets, one for the control processor and one for the VPs. Applications can be mapped to the VT architecture in a variety of ways but it is es- 


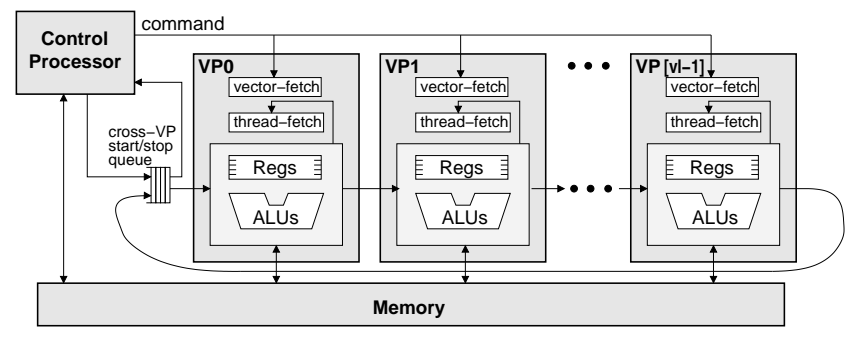

Figure 1: Abstract model of a vector-thread architecture. A control processor interacts with a virtual processor vector (an ordered sequence of VPs).

\begin{tabular}{|c|c|c|c|c|}
\hline & VP0 & VP1 & $\bullet \bullet$ & VP[vl-1] \\
\hline \multicolumn{5}{|l|}{ vector-fetch } \\
\hline & lb $r 0(r 1) \rightarrow r 4$ & lb $r 0(r 1) \rightarrow r 4$ & & lb $r 0(r 1) \rightarrow r 4$ \\
\hline & lb $r 0(r 2)->r 5$ & lb $r 0(r 2) \rightarrow r 5$ & & lb $r 0(r 2) \rightarrow r 5$ \\
\hline & add $r 4, r 5->r 6$ & add $r 4, r 5->r 6$ & & add $r 4, r 5->r 6$ \\
\hline & sb $r 6, r 0(r 3)$ & sb $r 6, r 0(r 3)$ & & sb $r 6, r 0(r 3)$ \\
\hline
\end{tabular}

Figure 2: Vector-fetch commands. For simple data-parallel loops, the control processor can use a vector-fetch command to send an atomic instruction block (AIB) to all the VPs in parallel. In this vector-vector add example, we assume that $r O$ has been loaded with each VP's index number; and $r 1, r 2$, and $r 3$ contain the base addresses of the input and output vectors. The instruction notation places the destination registers after the " $->$ ".

pecially well suited to executing loops; each VP executes a single iteration of the loop and the control processor is responsible for managing the execution.

A virtual processor contains a set of registers and has the ability to execute RISC-like instructions with virtual register specifiers. VP instructions are grouped into atomic instruction blocks (AIBs), the unit of work issued to a VP at one time. There is no automatic program counter or implicit instruction fetch mechanism for VPs; all instruction blocks must be explicitly requested by either the control processor or the VP itself.

The control processor can direct the VPs' execution using a vector-fetch command to issue an AIB to all the VPs in parallel, or a $V P$-fetch to target an individual VP. Vector-fetch commands provide a programming model similar to conventional vector machines except that a large block of instructions can be issued at once. As a simple example, Figure 2 shows the mapping for a dataparallel vector-vector add loop. The AIB for one iteration of the loop contains two loads, an add, and a store. A vector-fetch command sends this AIB to all the VPs in parallel and thus initiates $v 1$ loop iterations, where $\mathrm{vl}$ is the length of the VPV (i.e., the vector length). Every VP executes the same instructions but operates on distinct data elements as determined by its index number. As a more efficient alternative to the individual VP loads and stores shown in the example, a VT architecture can also provide vectormemory commands issued by the control processor which move a vector of elements between memory and one register in each VP.

The VT abstract model connects VPs in a unidirectional ring topology and allows a sending instruction on VP $(n)$ to transfer data directly to a receiving instruction on VP $(n+1)$. These cross$V P$ data transfers are dynamically scheduled and resolve when the data becomes available. Cross-VP data transfers allow loops with cross-iteration dependencies to be efficiently mapped to the vectorthread architecture, as shown by the example in Figure 3. A single vector-fetch command can introduce a chain of prevVP receives and nextVP sends that spans the VPV. The control processor can push an initial value into the cross-VP start/stop queue (shown in Figure 1) before executing the vector-fetch command. After the chain executes, the final cross-VP data value from the last VP wraps

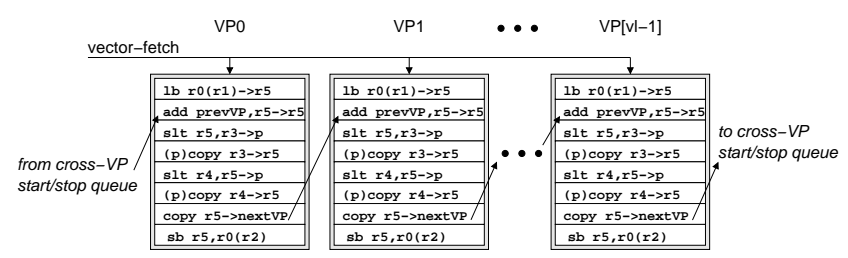

Figure 3: Cross-VP data transfers. For loops with cross-iteration dependencies, the control processor can vector-fetch an AIB that contains cross-VP data transfers. In this saturating parallel prefix sum example, we assume that $r 0$ has been loaded with each VP's index number, $r 1$ and $r 2$ contain the base addresses of the input and output vectors, and $r 3$ and $r 4$ contain the min and max values of the saturation range. The instruction notation uses " $(p)$ " to indicate predication.

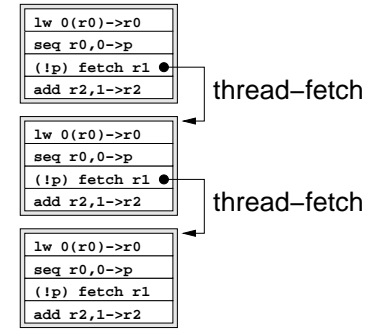

Figure 4: VP threads. Thread-fetches allow a VP to request its own AIBs and thereby direct its own control flow. In this pointer-chase example, we assume that $r 0$ contains a pointer to a linked list, $r 1$ contains the address of the AIB, and $r 2$ contains a count of the number of links traversed.

around and is written into this same queue. It can then be popped by the control processor or consumed by a subsequent prevVP receive on VP0 during stripmined loop execution.

The VT architecture also allows VPs to direct their own control flow. A VP executes a thread-fetch to request an AIB to execute after it completes its active AIB, as shown in Figure 4. Fetch instructions may be predicated to provide conditional branching. A VP thread persists as long as each AIB contains an executed fetch instruction, but halts once the VP stops issuing thread-fetches. Once a VP thread is launched, it executes to completion before the next command from the control processor takes effect. The control processor and VPs all operate concurrently in the same address space. Memory dependencies between these processors are preserved via explicit memory fence and synchronization operations or atomic read-modify-write operations.

The ability to freely intermix vector-fetches and thread-fetches allows a VT architecture to combine the best attributes of the vector and multithreaded execution paradigms. As shown in Figure 5, the control processor can issue a vector-fetch command to launch a vector of VP threads, each of which continues to execute as long as it issues thread-fetches. These thread-fetches break the rigid control flow of traditional vector machines, enabling the VP threads to follow independent control paths. Thread-fetches broaden the range of loops which can be mapped efficiently to VT, allowing the VPs to execute data-parallel loop iterations with conditionals or even inner-loops. Apart from loops, the VPs can also be used as free-running threads, where they operate independently from the control processor and retrieve tasks from a shared work queue.

The VT architecture allows software to efficiently expose structured parallelism and locality at a fine granularity. Compared to a conventional threaded architecture, the VT model allows common bookkeeping code to be factored out and executed once on the control processor rather than redundantly in each thread. AIBs enable a VT machine to efficiently amortize instruction fetch overhead, and provide a framework for cleanly handling temporary 


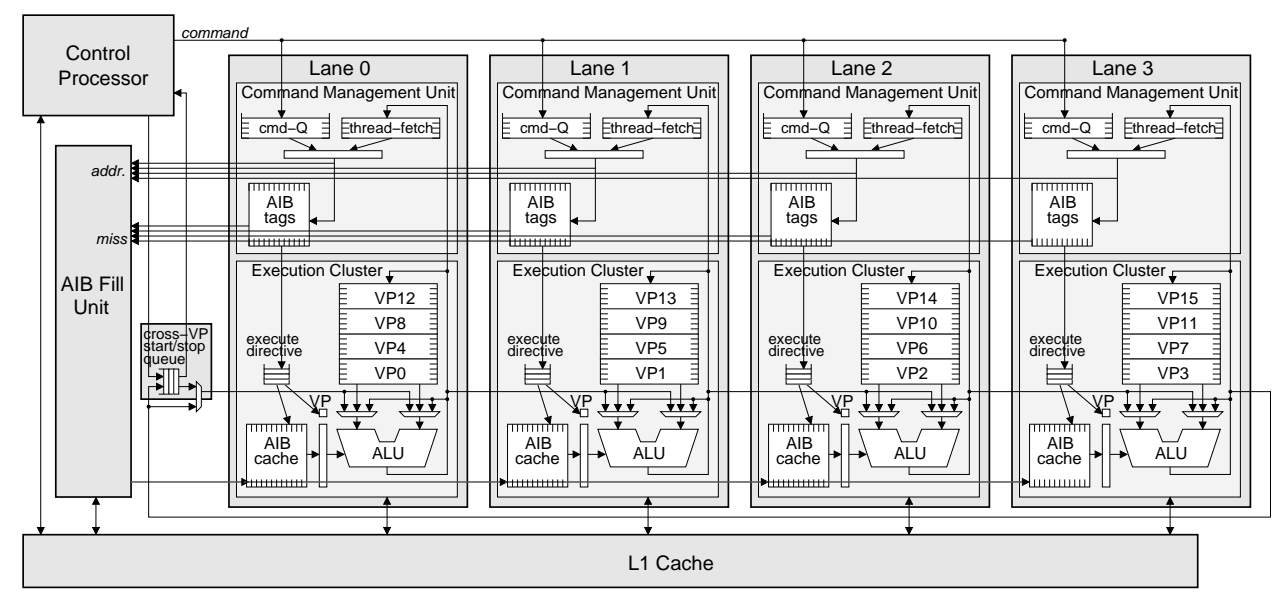

Figure 6: Physical model of a VT machine. The implementation shown has four parallel lanes in the vector-thread unit (VTU), and VPs are striped across the lane array with the low-order bits of a VP index indicating the lane to which it is mapped. The configuration shown uses VPs with five virtual registers, and with twenty physical registers each lane is able to support four VPs. Each lane is divided into a command management unit (CMU) and an execution cluster, and the execution cluster has an associated cross-VP start-stop queue.

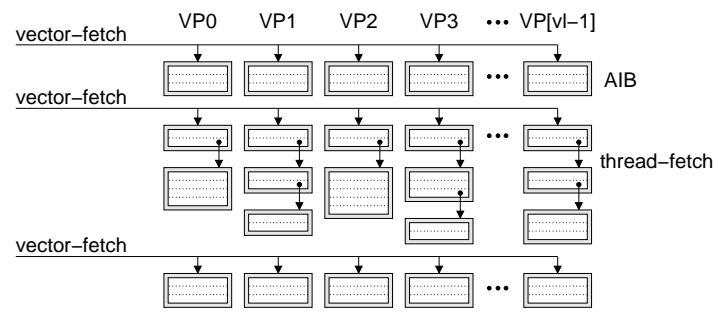

Figure 5: The control processor can use a vector-fetch command to send an AIB to all the VPs, after which each VP can use thread-fetches to fetch its own AIBs.

state. Vector-fetch commands explicitly encode parallelism and instruction locality, allowing a VT machine to attain high performance while amortizing control overhead. Vector-memory commands avoid separate load and store requests for each element, and can be used to exploit memory data-parallelism even in loops with non-data-parallel compute. For loops with cross-iteration dependencies, cross-VP data transfers explicitly encode fine-grain communication and synchronization, avoiding heavyweight interthread memory coherence and synchronization primitives.

\subsection{VT Physical Model}

An architectural paradigm's physical model is the expected structure for efficient implementations of the abstract model. The VT physical model contains a conventional scalar unit for the control processor together with a vector-thread unit (VTU) that executes the VP code. To exploit the parallelism exposed by the VT abstract model, the VTU contains a parallel array of processing lanes as shown in Figure 6. Lanes are the physical processors which VPs map onto, and the VPV is striped across the lane array. Each lane contains physical registers, which hold the state of VPs mapped to the lane, and functional units, which are time-multiplexed across the VPs. In contrast to traditional vector machines, the lanes in a VT machine execute decoupled from each other. Figure 7 shows an abstract view of how VP execution is time-multiplexed on the lanes for both vector-fetched and thread-fetched AIBs. This fine-grain interleaving helps VT machines hide functional unit, memory, and thread-fetch latencies.

As shown in Figure 6, each lane contains both a command management unit (CMU) and an execution cluster. An execution cluster consists of a register file, functional units, and a small AIB cache.

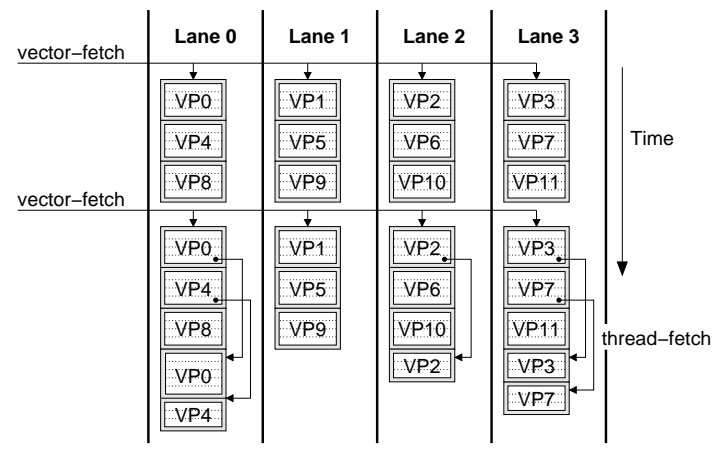

Figure 7: Lane Time-Multiplexing. Both vector-fetched and threadfetched AIBs are time-multiplexed on the physical lanes.

The lane's CMU buffers commands from the control processor in a queue (cmd-Q) and holds pending thread-fetch addresses for the lane's VPs. The CMU also holds the tags for the lane's AIB cache. The AIB cache can hold one or more AIBs and must be at least large enough to hold an AIB of the maximum size defined in the VT architecture.

The CMU chooses a vector-fetch, VP-fetch, or thread-fetch command to process. The fetch command contains an address which is looked up in the AIB tags. If there is a miss, a request is sent to the fill unit which retrieves the requested AIB from the primary cache. The fill unit handles one lane's AIB miss at a time, except if lanes are executing vector-fetch commands when refill overhead is amortized by broadcasting the AIB to all lanes simultaneously.

After a fetch command hits in the AIB cache or after a miss refill has been processed, the CMU generates an execute directive which contains an index into the AIB cache. For a vector-fetch command the execute directive indicates that the AIB should be executed by all VPs mapped to the lane, while for a VP-fetch or thread-fetch command it identifies a single VP to execute the AIB. The execute directive is sent to a queue in the execution cluster, leaving the CMU free to begin processing the next command. The CMU is able to overlap the AIB cache refill for new fetch commands with the execution of previous ones, but must track which AIBs have outstanding execute directives to avoid overwriting their entries in the AIB cache. The CMU must also ensure that the VP threads execute to completion before initiating a subsequent vector-fetch.

To process an execute directive, the cluster reads VP instructions 
one by one from the AIB cache and executes them for the appropriate VP. When processing an execute-directive from a vector-fetch command, all of the instructions in the AIB are executed for one VP before moving on to the next. The virtual register indices in the VP instructions are combined with the active VP number to create an index into the physical register file. To execute a fetch instruction, the cluster sends the requested AIB address to the CMU where the VP's associated pending thread-fetch register is updated.

The lanes in the VTU are inter-connected with a unidirectional ring network to implement the cross-VP data transfers. When a cluster encounters an instruction with a prevVP receive, it stalls until the data is available from its predecessor lane. When the VT architecture allows multiple cross-VP instructions in a single AIB, with some sends preceding some receives, the hardware implementation must provide sufficient buffering of send data to allow all the receives in an AIB to execute. By induction, deadlock is avoided if each lane ensures that its predecessor can never be blocked trying to send it cross-VP data.

\section{The SCALE VT Architecture}

SCALE is an instance of the VT architectural paradigm designed for embedded systems. The SCALE architecture has a MIPS-based control processor extended with a VTU. The SCALE VTU aims to provide high performance at low power for a wide range of applications while using only a small area. This section describes the SCALE VT architecture, presents a simple code example implemented on SCALE, and gives an overview of the SCALE microarchitecture and SCALE processor prototype.

\subsection{SCALE Extensions to VT}

\section{Clusters}

To improve performance while reducing area, energy and circuit delay, SCALE extends the single-cluster VT model (shown in Figure 1) by partitioning VPs into multiple execution clusters with independent register sets. VP instructions target an individual cluster and perform RISC-like operations. Source operands must be local to the cluster, but results can be written to any cluster in the VP, and an instruction can write its result to multiple destinations. Each cluster within a VP has a separate predicate register, and instructions can be positively or negatively predicated.

SCALE clusters are heterogeneous, but all clusters support basic integer operations. Cluster 0 additionally supports memory access instructions, cluster 1 supports fetch instructions, and cluster 3 supports integer multiply and divide. Though not used in this paper, the SCALE architecture allows clusters to be enhanced with layers of additional functionality (e.g., floating-point operations, fixed-point operations, and sub-word SIMD operations), or new clusters to be added to perform specialized operations.

\section{Registers and VP Configuration}

The general registers in each cluster of a VP are categorized as either private registers ( $\mathrm{pr}$ 's) and shared registers ( $\mathrm{sr}$ 's). Both private and shared registers can be read and written by VP instructions and by commands from the control processor. The main difference is that private registers preserve their values between AIBs, while shared registers may be overwritten by a different VP. Shared registers can be used as temporary state within an AIB to increase the number of VPs that can be supported by a fixed number of physical registers. The control processor can also vector-write the shared registers to broadcast scalar values and constants used by all VPs.

In addition to the general registers, each cluster also has programmer-visible chain registers ( $\mathrm{cr} 0$ and $\mathrm{cr} 1$ ) associated with the two ALU input operands. These can be used as sources and destinations to avoid reading and writing the register files. Like shared registers, chain registers may be overwritten between AIBs, and they are also implicitly overwritten when a VP instruction uses their associated operand position. Cluster 0 has a special chain register called the store-data ( $\mathrm{sd}$ ) register through which all data for VP stores must pass.

In the SCALE architecture, the control processor configures the VPs by indicating how many shared and private registers are required in each cluster. The length of the virtual processor vector changes with each re-configuration to reflect the maximum number of VPs that can be supported. This operation is typically done once outside each loop, and state in the VPs is undefined across reconfigurations. Within a lane, the VTU maps shared VP registers to shared physical registers. Control processor vector-writes to a shared register are broadcast to each lane, but individual VP writes to a shared register are not coherent across lanes, i.e., the shared registers are not global registers.

\section{Vector-Memory Commands}

In addition to VP load and store instructions, SCALE defines vector-memory commands issued by the control processor for efficient structured memory accesses. Like vector-fetch commands, these operate across the virtual processor vector; a vector-load writes the load data to a private register in each VP, while a vectorstore reads the store data from a private register in each VP. SCALE also supports vector-load commands which target shared registers to retrieve values used by all VPs. In addition to the typical unitstride and strided vector-memory access patterns, SCALE provides vector segment accesses where each VP loads or stores several contiguous memory elements to support "array-of-structures" data layouts efficiently.

\subsection{SCALE Code Example}

This section presents a simple code example to show how SCALE is programmed. The $\mathrm{C}$ code in Figure 8 implements a simplified version of the ADPCM speech decoder. Input is read from a unit-stride byte stream and output is written to a unit-stride halfword stream. The loop is non-vectorizable because it contains two loop-carried dependencies: the index and valpred variables are accumulated from one iteration to the next with saturation. The loop also contains two table lookups.

The SCALE code to implement the example decoder function is shown in Figure 9. The code is divided into two sections with MIPS control processor code in the . text section and SCALE VP code in the . sisa (SCALE ISA) section. The SCALE VP code implements one iteration of the loop with a single AIB; cluster 0 accesses memory, cluster 1 accumulates index, cluster 2 accumulates valpred, and cluster 3 does the multiply.

The control processor first configures the VPs using the vcfgvl command to indicate the register requirements for each cluster. In this example, $\mathrm{c} 0$ uses one private register to hold the input data and two shared registers to hold the table pointers; $\mathrm{c} 1$ and $\mathrm{c} 2$ each use three shared registers to hold the min and max saturation values and a temporary; c2 also uses a private register to hold the output value; and c 3 uses only chain registers so it does not need any shared or private registers. The configuration indirectly sets $\mathrm{vl}-$ max, the maximum vector length. In a SCALE implementation with 32 physical registers per cluster and four lanes, vlmax would be: $(\lfloor(32-3) / 1\rfloor) \times 4=116$, limited by the register demands of cluster 2 . The vcfgvl command also sets $v 1$, the active vectorlength, to the minimum of vlmax and the length argument provided; the resulting length is returned as a result. The control pro- 


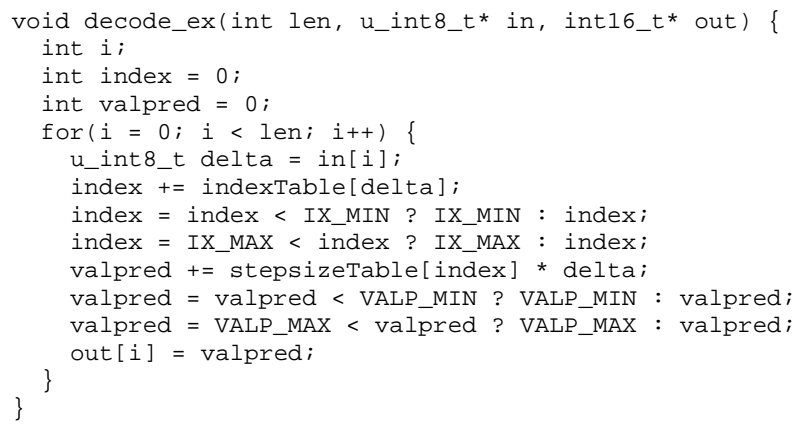

Figure 8: $\mathrm{C}$ code for decoder example.

cessor next vector-writes several shared VP registers with constants using the vwrsh command, then uses the xvppush command to push the initial index and valpred values into the cross-VP start/stop queues for clusters 1 and 2 .

The ISA for a VT architecture is defined so that code can be written to work with any number of VPs, allowing the same object code to run on implementations with varying or configurable resources. To manage the execution of the loop, the control processor uses stripmining to repeatedly launch a vector of loop iterations. For each iteration of the stripmine loop, the control processor uses the setvl command which sets the vectorlength to the minimum of vlmax and the length argument provided (i.e., the number of iterations remaining for the loop); the resulting vector-length is also returned as a result. In the decoder example, the control processor then loads the input using an auto-incrementing vector-load-byte-unsigned command ( $\mathrm{vl}-$ buai), vector-fetches the AIB to compute the decode, and stores the output using an auto-incrementing vector-store-halfword command (vshai). The cross-iteration dependencies are passed from one stripmine vector to the next through the cross-VP start/stop queues. At the end of the function the control processor uses the xvppop command to pop and discard the final values.

The SCALE VP code implements one iteration of the loop in a straightforward manner with no cross-iteration static scheduling. Cluster 0 holds the delta input value in pro from the previous vector-load. It uses a VP load to perform the indexTable lookup and sends the result to cluster 1 . Cluster 1 uses five instructions to accumulate and saturate index, using prevVP and nextVP to receive and send the cross-iteration value, and the psel (predicateselect) instruction to optimize the saturation. Cluster 0 then performs the stepsizeTable lookup using the index value, and sends the result to cluster 3 where it is multiplied by delta. Cluster 2 uses five instructions to accumulate and saturate valpred, writing the result to pro for the subsequent vector-store.

\subsection{SCALE Microarchitecture}

The SCALE microarchitecture is an extension of the general VT physical model shown in Figure 6. A lane has a single CMU and one physical execution cluster per VP cluster. Each cluster has a dedicated output bus which broadcasts data to the other clusters in the lane, and it also connects to its sibling clusters in neighboring lanes to support cross-VP data transfers. An overview of the SCALE lane microarchitecture is shown in Figure 10.

\section{Micro-Ops and Cluster Decoupling}

The SCALE software ISA is portable across multiple SCALE implementations, but is designed to be easy to translate into implementation-specific micro-operations, or micro-ops. The assembler translates the SCALE software ISA into the native hard- .text \# MIPS control processor code

decode_ex: \# a0=len, a1=in, a $2=$ out

\# configure VPs: $c 0: p, s$ c1:p, s c2:p, s c3:p, s

vcfgvi t1, a0, $1,2,0,3,1,3,0,0$

$\#(\mathrm{v} l, \mathrm{t} 1)=\min (\mathrm{a} 0, \mathrm{vlmax})$

sll t1, t1, 1 \# output stride

la to, indextable

vwrsh to, c0/sro \# indextable addr.

la to, stepsizetable

vwrsh to, c0/sr1 \# stepsizeTable addr.

vwrsh IX_MIN, c1/sro \# index min

vwrsh IX_MAX, c1/sr1 \# index max

Vwrsh VALP MIN, c2/sr0\# valpred min

vwrsh VALP MAX, c2/sr1\# valpred max

xvppush $\$ 0, \overline{c 1}$ \# push initial index $=0$

xvppush $\$ 0, \mathrm{c} 2 \quad$ \# push initial valpred $=0$

stripmineloop:

setvl $t 2, a 0 \quad \#(v l, t 2)=\min (a 0, v l \max )$

vlbuai a1, t2, c0/pro \# vector-load input, inc ptr

vf vtu_decode_ex \# vector-fetch AIB

vshai a2, t1, c2/pr0 \# vector-store output, inc ptr

subu a0, t2 \# decrement count

bnez a0, stripmineloop \# loop until done

xvppop \$0, c1 \# pop final index, discard

xvppop $\$ 0$, c2 \# pop final valpred, discard

vsync \# wait until VPs are done

jr ra

\# return

. sisa \# SCALE VP code

vtu_decode_ex:

-aib begin

c0 sll pro, $2 \rightarrow \mathrm{cr}$

c0 lw $\mathrm{cr} 1(\mathrm{sr} 0) \rightarrow \mathrm{c} 1 / \mathrm{cr} 0$

c0 copy pro $\rightarrow \mathrm{c} 3 / \mathrm{cr} 0$

c1 addu cr0, prevVP $\rightarrow$ cr0

c1 slt cro, sro $\rightarrow p$

c1 psel cr0, sro $\rightarrow$ sr2

c1 slt sr1, sr2 $\rightarrow \mathrm{p}$

c1 psel sr2, sr1 $\rightarrow$ c0/cr0, nextVP

c0 sll cr0, $2 \rightarrow \mathrm{cr} 1$

$\mathrm{c} 0 \mathrm{lw} \quad \mathrm{cr} 1(\mathrm{sr} 1) \rightarrow \mathrm{c} 3 / \mathrm{cr}$

c3 mult.1o cr0, cr1 $\rightarrow$ c2/cr0

c2 addu cr0, prevVP $\rightarrow$ cr0

c2 slt cro, sro $\rightarrow p$

c2 psel cr0, sro $\rightarrow$ sr2

c2 slt sr1, sr2 $\rightarrow$ p

c2 psel sr2, sr1 $\rightarrow$ pro, nextVP

.aib end

\# word offset

\# load index

\# copy delta

\# accum. index

\# index min

\# index min

\# index max

\# index max

\# word offset

\# load step

\# step*delta

\# accum. valpred

\# valpred min

\# valpred min

\# valpred max

\# valpred max

Figure 9: SCALE code implementing decoder example from Figure 8.

ware ISA at compile time. There are three categories of hardware micro-ops: a compute-op performs the main RISC-like operation of a VP instruction; a transport-op sends data to another cluster; and, a writeback-op receives data sent from an external cluster. The assembler reorganizes micro-ops derived from an AIB into micro-op bundles which target a single cluster and do not access other clusters' registers. Figure 11 shows how the SCALE VP instructions from the decoder example are translated into micro-op bundles. All inter-cluster data dependencies are encoded by the transportops and writeback-ops which are added to the sending and receiving cluster respectively. This allows the micro-op bundles for each cluster to be packed together independently from the micro-op bundles for other clusters.

Partitioning inter-cluster data transfers into separate transport and writeback operations enables decoupled execution between clusters. In SCALE, a cluster's AIB cache contains micro-op bundles, and each cluster has a local execute directive queue and local control. Each cluster processes its transport-ops in order, broadcasting result values onto its dedicated output data bus; and each cluster processes its writeback-ops in order, writing the values from external clusters to its local registers. The inter-cluster data dependencies are synchronized with handshake signals which extend between the clusters, and a transaction only completes when both the 


\begin{tabular}{|c|c|c|c|c|c|c|c|c|c|c|c|}
\hline \multicolumn{3}{|c|}{ Cluster 0} & \multicolumn{3}{|c|}{ Cluster 1} & \multicolumn{3}{|c|}{ Cluster 2} & \multicolumn{3}{|c|}{ Cluster 3} \\
\hline wb-op & compute-op & tp-op & wb-op & compute-op & tp-op & wb-op & compute-op & tp-op & wb-op & compute-op & tp-op \\
\hline & sll pr0,2 $\rightarrow$ cr1 & & $\overline{b, \mathrm{c} 0 \rightarrow \mathrm{cr} 0}$ & addu $\quad \mathrm{cr} 0, \mathrm{pVP} \rightarrow \mathrm{cr} 0$ & & $\mid b, c 3 \rightarrow c r 0$ & $\mathrm{addu} \quad \mathrm{cr} 0, \mathrm{pVP} \rightarrow \mathrm{cr} 0$ & & $b, \mathrm{c} 0 \rightarrow \mathrm{cr} 0$ & & \\
\hline & $1 \mathrm{w} \operatorname{cr} 1(\operatorname{sr} 0)$ & $\rightarrow \mathrm{c} 1$ & & slt cr0, sro $\rightarrow$ p & & & slt cr0, sro $\rightarrow p$ & & $b, \mathrm{c} 0 \rightarrow \mathrm{cr} 1$ & mult cr0, cr1 & $\rightarrow c 2$ \\
\hline $\mathrm{c} 1 \rightarrow \mathrm{cr} 0$ & copy pro & $\rightarrow c 3$ & & psel cr0, sro $\rightarrow$ sr2 & & & psel cr0, sr0 $\rightarrow$ sr2 & & & & \\
\hline & sll cr $0,2 \rightarrow \operatorname{cr} 1$ & & & slt sr 1, sr $2 \rightarrow p$ & & & slt $\operatorname{sr} 1$, sr $2 \rightarrow p$ & & & & \\
\hline & $1 \mathrm{w} \operatorname{crl}(\mathrm{sr} 1)$ & $\rightarrow c 3$ & & psel sr2,sr1 & $\rightarrow \mathrm{nVP}, \mathrm{c} 0$ & & psel sr2, sr1 $\rightarrow$ pro & $\rightarrow \mathrm{nVP}$ & & & \\
\hline
\end{tabular}

Figure 11: Cluster micro-op bundles for the AIB in Figure 9. The writeback-op field is labeled as 'wb-op' and the transport-op field is labeled as 'tp-op'. A writeback-op is marked with ' $b$ ' when the dependency order is writeback-op followed by compute-op. The prevVP and nextVP identifiers are abbreviated as 'pVP' and 'nVP'.

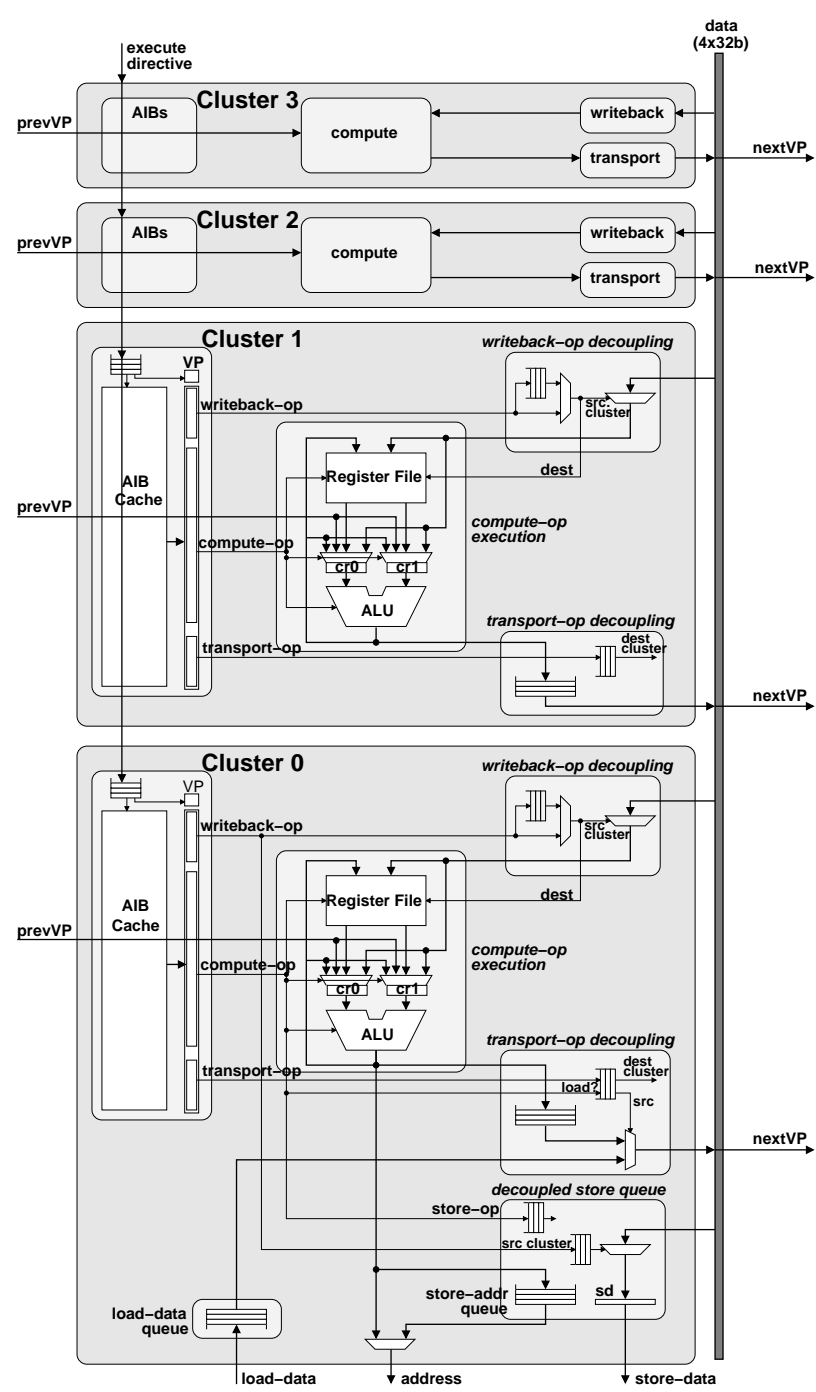

Figure 10: SCALE Lane Microarchitecture. The AIB caches in SCALE hold micro-op bundles. The compute-op is a local RISC operation on the cluster, the transport-op sends data to external clusters, and the writeback-op receives data from external clusters. Clusters 1,2 , and 3 are basic cluster designs with writeback-op and transport-op decoupling resources (cluster 1 is shown in detail, clusters 2 and 3 are shown in abstract). Cluster 0 connects to memory and includes memory access decoupling resources.

sender and the receiver are ready. Although compute-ops execute in order, each cluster contains a transport queue to allow execution to proceed without waiting for external destination clusters to receive the data, and a writeback queue to allow execution to proceed without waiting for data from external clusters (until it is needed by a compute-op). These queues make inter-cluster synchroniza-

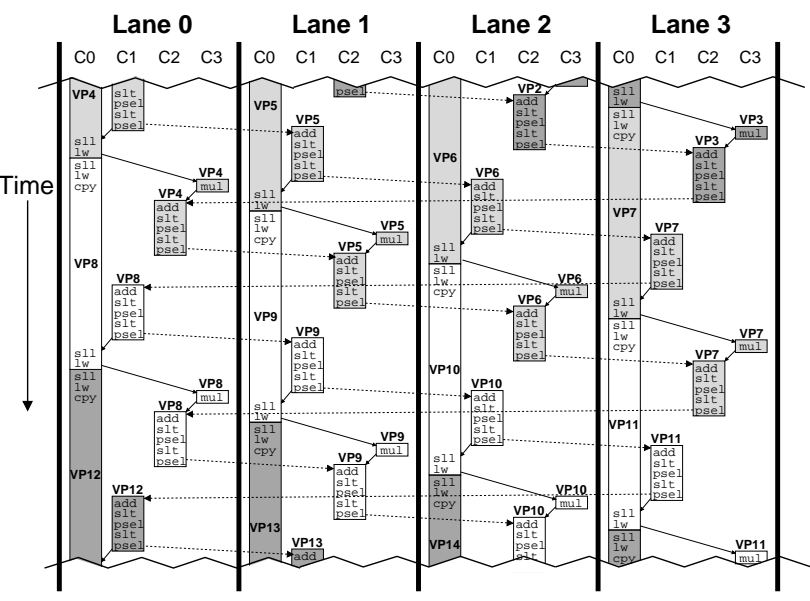

Figure 12: Execution of decoder example on SCALE. Each cluster executes in-order, but cluster and lane decoupling allows the execution to automatically adapt to the software critical path. Critical dependencies are shown with arrows (solid for inter-cluster within a lane, dotted for cross-VP).

tion more flexible, and thereby enhance cluster decoupling.

A schematic diagram of the example decoder loop executing on SCALE (extracted from simulation trace output) is shown in Figure 12. Each cluster executes the vector-fetched AIB for each VP mapped to its lane, and decoupling allows each cluster to advance to the next VP independently. Execution automatically adapts to the software critical path as each cluster's local data dependencies resolve. In the example loop, the accumulations of index and valpred must execute serially, but all of the other instructions are not on the software critical path. Furthermore, the two accumulations can execute in parallel, so the cross-iteration serialization penalty is paid only once. Each loop iteration (i.e., VP) executes over a period of 30 cycles, but the combination of multiple lanes and cluster decoupling within each lane leads to as many as six loop iterations executing simultaneously.

\section{Memory Access Decoupling}

All VP loads and stores execute on cluster 0 (c0), and it is specially designed to enable access-execute decoupling [11]. Typically, c0 loads data values from memory and sends them to other clusters, computation is performed on the data, and results are returned to $\mathrm{c} 0$ and stored to memory. With basic cluster decoupling, $\mathrm{c} 0$ can continue execution after a load without waiting for the other clusters to receive the data. Cluster 0 is further enhanced to hide memory latencies by continuing execution after a load misses in the cache, and therefore it may retrieve load data from the cache out of order. However, like other instructions, load operations on cluster 0 use transport-ops to deliver data to other clusters in order, and $\mathrm{c} 0$ uses a load data queue to buffer the data and preserve the correct ordering.

Importantly, when cluster 0 encounters a store, it does not stall to 


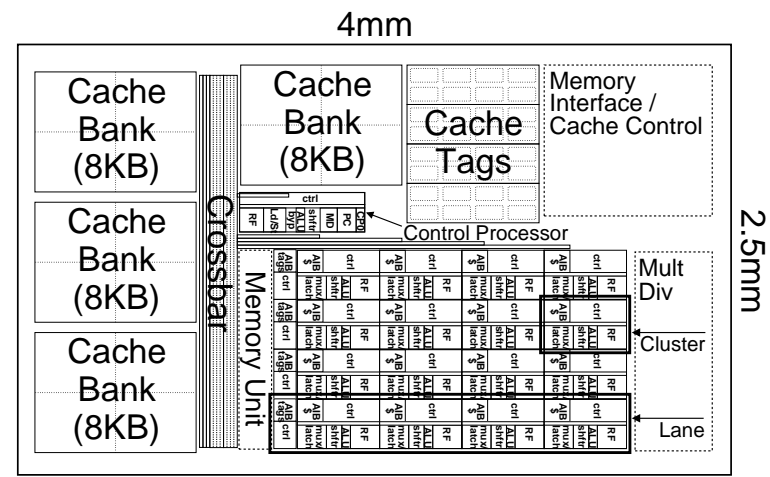

Figure 13: Preliminary floorplan estimate for SCALE prototype. The prototype contains a scalar control processor, four 32-bit lanes with four execution clusters each, and $32 \mathrm{~KB}$ of cache in an estimated $10 \mathrm{~mm}^{2}$ in $0.18 \mu \mathrm{m}$ technology.

wait for the data to be ready. Instead it buffers the store operation, including the store address, in the decoupled store queue until the store data is available. When a SCALE VP instruction targets the $\mathrm{sd}$ register, the resulting transport-op sends data to the store unit rather than to $\mathrm{c} 0$; thus, the store unit acts as a primary destination for inter-cluster transport operations and it handles the writebackops for sd. Store decoupling allows a lane's load stream to slip ahead of its store stream, but loads for a given VP are not allowed to bypass previous stores to the same address by the same VP.

\section{Vector-Memory Accesses}

Vector-memory commands are sent to the clusters as special execute directives which generate micro-ops instead of reading them from the AIB cache. For a vector-load, writeback-ops on the destination cluster receive the load data; and for a vector-store, computeops and transport-ops on the source cluster read and send the store data. Chaining is provided to allow overlapped execution of vectorfetched AIBs and vector-memory operations.

The vector-memory commands are also sent to the vectormemory unit which performs the necessary cache accesses. The vector-memory unit can only send one address to the cache each cycle, but it takes advantage of the structured access patterns to load or store multiple elements with each access. The vector-memory unit communicates load and store data to and from cluster 0 in each lane to reuse the buffering already provided for the decoupled VP loads and stores.

\subsection{Prototype}

We are currently designing a prototype SCALE processor, and an initial floorplan is shown in Figure 13. The prototype contains a single-issue MIPS scalar control processor, four 32-bit lanes with four execution clusters each, and a $32 \mathrm{~KB}$ shared primary cache. The VTU has 32 registers per cluster and supports up to 128 virtual processors. The prototype's unified L1 cache is 32-way setassociative [15] and divided into four banks. The vector memory unit can perform a single access per cycle, fetching up to 128 bits from a single bank, or all lanes can perform VP accesses from different banks. The cache is non-blocking and connects to off-chip DDR2 SDRAM.

The area estimate of around $10 \mathrm{~mm}^{2}$ in $0.18 \mu \mathrm{m}$ technology is based on microarchitecture-level datapath designs for the control processor and VTU lanes; cell dimensions based on layout for the datapath blocks, register files, CAMs, SRAM arrays, and crossbars; and estimates for the synthesized control logic and external interface overhead. We have designed the SCALE prototype to

\begin{tabular}{|l|l|}
\hline \multicolumn{2}{|c|}{ Vector-Thread Unit } \\
\hline Number of lanes & 4 \\
Clusters per lane & 4 \\
Registers per cluster & 32 \\
AIB cache uops per cluster & 32 \\
Intra-cluster bypass latency & 0 cycles \\
Intra-lane transport latency & 1 cycle \\
Cross-VP transport latency & 0 cycles \\
Clock frequency & $400 \mathrm{MHz}$ \\
\hline \hline \multicolumn{2}{|c|}{ L1 Unified Cache } \\
\hline Size & $32 \mathrm{~KB}$ \\
Associativity & 32 (CAM tags) \\
Line size & $32 \mathrm{~B}$ \\
Banks & 4 \\
Maximum bank access width & $16 \mathrm{~B}$ \\
Store miss policy & write-allocate/write-back \\
Load-use latency & 2 cycles \\
\hline \hline \multicolumn{2}{|c|}{ Memory System } \\
\hline DRAM type & DDR2 \\
Data bus width & 64 bits \\
DRAM clock frequency & $200 \mathrm{MHz}$ \\
Data bus frequency & $400 \mathrm{MHz}$ \\
Minimum load-use latency & 35 processor cycles \\
\hline
\end{tabular}

Table 1: Default parameters for SCALE simulations.

fit into a compact area to reduce wire delays and design complexity, and to support tiling of multiple SCALE processors on a CMP for increased processing throughput. The clock frequency target is $400 \mathrm{MHz}$ based on a $25 \mathrm{FO} 4$ cycle time, chosen as a compromise between performance, power, and design complexity.

\section{Evaluation}

This section contains an evaluation and analysis of SCALE running a diverse selection of embedded benchmark codes. We first describe the simulation methodology and benchmarks, then discuss how the benchmark codes were mapped to the VT architecture and the resulting efficiency of execution.

\subsection{Programming and Simulation Methodology}

SCALE was designed to be compiler-friendly, and a C compiler is under development. For the results in this paper, all VTU code was hand-written in SCALE assembler (as in Figure 9) and linked with $\mathrm{C}$ code compiled for the MIPS control processor using gCC. The same binary code was used across all SCALE configurations.

A detailed cycle-level, execution-driven microarchitectural simulator has been developed based on the prototype design. Details modeled in the VTU simulation include cluster execution of micro-ops governed by execute-directives; cluster decoupling and dynamic inter-cluster data dependency resolution; memory access decoupling; operation of the vector-memory unit; operation of the command management unit, including vector-fetch and threadfetch commands with AIB tag-checking and miss handling; and the AIB fill unit and its contention for the primary cache.

The VTU simulation is complemented by a cycle-based memory system simulation which models the multi-requester, multibanked, non-blocking, highly-associative CAM-based cache and a detailed memory controller and DRAM model. The cache accurately models bank conflicts between different requesters; exerts back-pressure in response to cache contention; tracks pending misses and merges in new requests; and models cache-line refills and writebacks. The DRAM simulation is based on the DDR2 chips used in the prototype design, and models a 64-bit wide memory port clocked at $200 \mathrm{MHz}(400 \mathrm{Mb} / \mathrm{s} /$ pin) including page refresh, precharge and burst effects.

The default simulation parameters are based on the prototype and are summarized in Table 1. An intra-lane transport from one cluster to another has a latency of one cycle (i.e. there will be a one cycle 
bubble between the producing instruction and the dependent instruction). Cross-VP transports are able to have zero cycle latency because the clusters are physically closer together and there is less fan-in for the receive operation. Cache accesses have a two cycle latency (two bubble cycles between load and use), and accesses which miss in the cache have a minimum latency of 35 cycles.

To show scaling effects, we model four SCALE configurations with one, two, four, and eight lanes. The one, two, and four lane configurations each include four cache banks and one 64-bit DRAM port. For eight lanes, the memory system was doubled to eight cache banks and two 64-bit memory ports to appropriately match the increased compute bandwidth.

\subsection{Benchmarks and Results}

We have implemented a selection of benchmarks (Table 2) to illustrate the key features of SCALE, including examples from network processing, image processing, cryptography, and audio processing. The majority of these benchmarks come from the EEMBC benchmark suite. The EEMBC benchmarks may either be run "outof-the-box" (OTB) as compiled unmodified C code, or they may be optimized (OPT) using assembly coding and arbitrary hand-tuning. This enables a direct comparison of SCALE running hand-written assembly code to optimized results from industry processors. Although OPT results match the typical way in which these processors are used, one drawback of this form of evaluation is that performance depends greatly on programmer effort, especially as EEMBC permits algorithmic and data-structure changes to many of the benchmark kernels, and optimizations used for the reported results are often unpublished. Also, not all of the EEMBC results are available for all processors, as results are often submitted for only one of the domain-specific suites (e.g., telecom).

We made algorithmic changes to several of the EEMBC benchmarks: rotate blocks the algorithm to enable rotating an 8-bit block completely in registers, pkt f low implements the packet descriptor queue using an array instead of a linked list, fir optimizes the default algorithm to avoid copying and exploit reuse, fbital uses a binary search to optimize the bit allocation, conven uses bit packed input data to enable multiple bit-level operations to be performed in parallel, and $\mathrm{f} f \mathrm{t}$ uses a radix-2 hybrid Stockham algorithm to eliminate bit-reversal and increase vector lengths.

Figure 14 shows the simulated performance of the various SCALE processor configurations relative to several reasonable competitors from among those with the best published EEMBC benchmark scores in each domain. For each of the different benchmarks, Table 3 shows VP configuration and vector-length statistics, and Tables 4 and 5 give statistics showing the effectiveness of the SCALE control and data hierarchies. These are discussed further in the following sections.

The AMD Au1100 was included to validate the SCALE control processor OTB performance, as it has a similar structure and clock frequency, and also uses gcc. The Philips TriMedia TM 1300 is a five-issue VLIW processor with a 32-bit datapath. It runs at $166 \mathrm{MHz}$ and has a $32 \mathrm{~KB} \mathrm{L1}$ instruction cache and $16 \mathrm{~KB} \mathrm{L1}$ data cache, with a 32-bit memory port running at $125 \mathrm{MHz}$. The Motorola PowerPC (MPC7447) is a four-issue out-of-order superscalar processor which runs at $1.3 \mathrm{GHz}$ and has $32 \mathrm{~KB}$ separate L1 instruction and data caches, a $512 \mathrm{~KB}$ L2 cache, and a 64-bit memory port running at $133 \mathrm{MHz}$. The OPT results for the processor use its Altivec SIMD unit which has a 128-bit datapath and four execution units. The VIRAM processor [4] is a research vector processor with four 64-bit lanes. VIRAM runs at $200 \mathrm{MHz}$ and includes $13 \mathrm{MB}$ of embedded DRAM supporting up to 256 bits each of load and store data, and four independent addresses per cycle.
The BOPS Manta is a clustered VLIW DSP with four clusters each capable of executing up to five instructions per cycle on 64-bit datapaths. The Manta 2.0 runs at $136 \mathrm{MHz}$ with $128 \mathrm{~KB}$ of on-chip memory connected to a 32-bit memory port running at $136 \mathrm{MHz}$. The TI TMS TMS320C6416 is a clustered VLIW DSP with two clusters each capable of executing up to four instructions per cycle. It runs at $720 \mathrm{MHz}$ and has a $16 \mathrm{~KB}$ instruction cache and a $16 \mathrm{~KB}$ data cache together with $1 \mathrm{MB}$ of on-chip SRAM. The TI has a 64bit memory interface running at $720 \mathrm{MHz}$. Apart from the Au1100 and SCALE, all other processors implement SIMD operations on packed subword values and these are widely exploited throughout the benchmark set.

Overall, the results show that SCALE can flexibly provide competitive performance with larger and more complex processors on a wide range of codes from different domains, and that performance generally scales well when adding new lanes. The results also illustrate the large speedups possible when algorithms are extensively tuned for a highly parallel processor versus compiled from standard reference code. SCALE results for $f f t$ and viterbi are not as competitive with the DSPs. This is partly due to these being preliminary versions of the code with further scope for tuning (e.g., moving the current radix-2 FFT to radix-4 and using outerloop vectorization for viterbi) and partly due to the DSPs having special support for these operations (e.g., complex multiply on BOPS). We expect SCALE performance to increase significantly with the addition of subword operations and with improvements to the microarchitecture driven by these early results.

\subsection{Mapping Parallelism to SCALE}

The SCALE VT architecture allows software to explicitly encode the parallelism and locality available in an application. This section evaluates the architecture's expressiveness in mapping different types of code.

\section{Data-Parallel Loops with No Control Flow}

Data-parallel loops with no internal control flow, i.e. simple vectorizable loops, may be ported to the VT architecture in a similar manner as other vector architectures. Vector-fetch commands encode the cross-iteration parallelism between blocks of instructions, while vector-memory commands encode data locality and enable optimized memory access. The EEMBC image processing benchmarks (rgbcmy, rgbyiq, hpg) are examples of streaming vectorizable code for which SCALE is able to achieve high performance that scales with the number of lanes in the VTU. A 4-lane SCALE achieves performance competitive with VIRAM for rgbyiq and rgbcmy despite having half the main memory bandwidth, primarily because VIRAM is limited by strided accesses while SCALE refills the cache with unit-stride bursts and then has higher strided bandwidth into the cache. For the unit-stride hpg benchmark, performance follows memory bandwidth with the 8lane SCALE approximately matching VIRAM.

\section{Data-Parallel Loops with Conditionals}

Traditional vector machines handle conditional code with predication (masking), but the VT architecture adds the ability to conditionally branch. Predication can be less overhead for small conditionals, but branching results in less work when conditional blocks are large. EEMBC dither is an example of a large conditional block in a data parallel loop. This benchmark converts a grey-scale image to black and white, and the dithering algorithm handles white pixels as a special case. In the SCALE code, each VP executes a conditional fetch for each pixel, executing only 18 SCALE instructions for white pixels versus 49 for non-white pixels. 


\begin{tabular}{|c|c|c|c|c|c|c|c|c|c|c|c|c|c|c|c|c|}
\hline \multirow{2}{*}{\multicolumn{2}{|c|}{$\begin{array}{l}\text { EEMBC } \\
\text { Benchmarks }\end{array}$}} & \multirow{3}{*}{$\begin{array}{l}\text { Data } \\
\text { Set } \\
- \\
\end{array}$} & \multirow{3}{*}{$\begin{array}{r}\text { OTB } \\
\text { Itr/Sec } \\
126\end{array}$} & \multirow{3}{*}{$\begin{array}{r}\begin{array}{r}\text { OPT } \\
\text { Itr/Sec }\end{array} \\
1505\end{array}$} & \multirow{3}{*}{\begin{tabular}{|r|}
$\begin{array}{r}\text { Kernel } \\
\text { Speedup }\end{array}$ \\
11.9 \\
\end{tabular}} & \multirow{3}{*}{$\begin{array}{r}\begin{array}{r}\text { Ops/ } \\
\text { Cycle }\end{array} \\
6.1 \\
\end{array}$} & \multirow{2}{*}{\begin{tabular}{|r|r} 
Mem B/ \\
Cycle
\end{tabular}} & \multicolumn{6}{|c|}{ Loop Type } & \multirow{2}{*}{\multicolumn{2}{|c|}{\begin{tabular}{|c|c|} 
Mem \\
VM & VP \\
\end{tabular}}} & \multirow[b]{2}{*}{ Description } \\
\hline & & & & & & & & DP & DC & $\mathbf{X I}$ & DI & DE & FT & & & \\
\hline rgbcmy & consumer & & & & & & 3.2 & $\bar{x}$ & & & & & & & $\bar{x}$ & RGB to CMYK color conversion \\
\hline rgbyiq & consumer & - & 56 & 1777 & 31.7 & 9.9 & 3.1 & $\bar{x}$ & & & & & & & $\bar{x}$ & RGB to YIQ color conversion \\
\hline hpg & consumer & - & 108 & 3317 & 30.6 & 9.5 & 2.0 & $\bar{x}$ & & & & & & $x$ & $x$ & High pass grey-scale filter \\
\hline text & office & - & 299 & 435 & 1.5 & 0.3 & 0.0 & & & & & $\bar{x}$ & & $\bar{x}$ & & Printer language parsing \\
\hline dither & office & - & 149 & 653 & 4.4 & 5.0 & 0.2 & $\bar{x}$ & $\bar{x}$ & & & & & $\bar{x}$ & $\bar{x}$ & Floyd-Steinberg grey-scale dithering \\
\hline rotate & office & $\overline{-}$ & 704 & 10112 & 14.4 & 7.5 & 0.0 & $\bar{x}$ & & & & & & $\bar{x}$ & $\bar{x}$ & Binary image 90 degree rotation \\
\hline lookup & network & - & 1663 & 8850 & 5.3 & 6.3 & 0.0 & & & & $x$ & & & $\bar{x}$ & $\bar{x}$ & IP route lookup using Patricia Trie \\
\hline pktflow & network & $\begin{array}{l}512 \mathrm{~KB} \\
1 \mathrm{MB} \\
2 \mathrm{MB}\end{array}$ & $\begin{array}{l}6694 \\
2330 \\
1189\end{array}$ & $\begin{array}{r}127677 \\
25609 \\
13473\end{array}$ & $\begin{array}{l}19.1 \\
11.0 \\
11.3\end{array}$ & $\begin{array}{l}7.8 \\
3.0 \\
3.1\end{array}$ & $\begin{array}{l}0.6 \\
3.6 \\
3.7\end{array}$ & & $x$ & $x$ & & & & $x$ & $x$ & IP packet processing \\
\hline pntrch & auto & - & 8771 & 38744 & 4.4 & 2.3 & 0.0 & & & & & & $\bar{x}$ & & $\times$ & Pointer chasing, searching linked list \\
\hline fir & auto & - & 56724 & 6105006 & 107.6 & 8.7 & 0.3 & $\bar{x}$ & & & & & & $\bar{x}$ & & Finite impulse response filter \\
\hline fbital & telecom & $\begin{array}{l}\text { typ } \\
\text { step } \\
\text { pent }\end{array}$ & $\begin{array}{r}860 \\
12523 \\
1304\end{array}$ & $\begin{array}{r}20897 \\
281938 \\
60958\end{array}$ & \begin{tabular}{l|}
24.3 \\
22.5 \\
46.7
\end{tabular} & $\begin{array}{l}4.0 \\
2.5 \\
3.6\end{array}$ & $\begin{array}{l}0.0 \\
0.0 \\
0.0\end{array}$ & & $\times$ & $\times$ & & & & $\times$ & $\times$ & Bit allocation for DSL modems \\
\hline $\mathrm{fft}$ & telecom & all & 6572 & 89713 & 13.6 & 6.1 & 0.0 & $\bar{x}$ & & & & & & $\bar{x}$ & & 256-pt fixed-point complex FFT \\
\hline autocor & telecom & $\begin{array}{l}\text { data1 } \\
\text { data2 } \\
\text { data3 }\end{array}$ & $\begin{array}{r}279339 \\
1888 \\
1980\end{array}$ & $\begin{array}{r}3131115 \\
64148 \\
78751\end{array}$ & $\begin{array}{l}11.2 \\
34.0 \\
39.8\end{array}$ & $\begin{array}{r}4.8 \\
11.2 \\
13.0\end{array}$ & $\begin{array}{l}0.2 \\
0.0 \\
0.0\end{array}$ & $x$ & & & & & & $x$ & & Fixed-point autocorrelation \\
\hline conven & telecom & $\begin{array}{l}\text { data1 } \\
\text { data2 } \\
\text { data3 }\end{array}$ & \begin{tabular}{l|}
2899 \\
3361 \\
4259 \\
\end{tabular} & $\begin{array}{l}2447980 \\
3085229 \\
3703703\end{array}$ & $\begin{array}{l}844.3 \\
917.8 \\
869.4\end{array}$ & $\begin{array}{r}9.8 \\
10.4 \\
9.5 \\
\end{array}$ & \begin{tabular}{l|}
0.0 \\
0.0 \\
0.1
\end{tabular} & $x$ & & & & & & $x$ & $x$ & Convolutional encoder \\
\hline $\begin{array}{l}\text { Other } \\
\text { Benchmark }\end{array}$ & & $\begin{array}{l}\text { Data } \\
\text { Set }\end{array}$ & $\begin{array}{r}\text { OTB Total } \\
\text { Cycles } \\
\end{array}$ & \begin{tabular}{|r|} 
OPT Total \\
Cycles \\
\end{tabular} & $\begin{array}{r}\text { Kernel } \\
\text { Speedup }\end{array}$ & $\begin{array}{r}\text { Ops/ } \\
\text { Cycle } \\
\end{array}$ & $\begin{array}{r}\text { Mem B/ } \\
\text { Cycle }\end{array}$ & DP & DC & $\begin{array}{l}\text { Loop } \\
\text { |XI }\end{array}$ & $\begin{array}{l}\text { Type } \\
\text { DI } \\
\end{array}$ & DE & FT & $\begin{array}{r}\mathbf{M} \\
\mathbf{V M} \\
\end{array}$ & VP & Description \\
\hline rijndael & MiBench & large & $420.8 \mathrm{M}$ & $219.0 \mathrm{M}$ & 2.4 & 2.5 & 0.0 & $\times$ & & & & & & $\times$ & $\times$ & Advanced Encryption Standard \\
\hline sha & MiBench & large & $141.3 \mathrm{M}$ & $64.8 \mathrm{M}$ & 2.2 & 1.8 & 0.0 & $\bar{x}$ & & $\bar{x}$ & & & & $x$ & $x$ & Secure hash algorithm \\
\hline qsort & MiBench & small & $35.0 \mathrm{M}$ & $21.4 \mathrm{M}$ & 3.5 & 2.3 & 2.7 & & & & & & $\bar{x}$ & & $x$ & Quick sort of strings \\
\hline adpcm_enc & Mediabench & - & $7.7 \mathrm{M}$ & $4.3 \mathrm{M}$ & 1.8 & 2.3 & 0.0 & & & $\times$ & & & & $\times$ & $x$ & Adaptive Differential PCM encode \\
\hline adpcm_dec & Mediabench & - & $6.3 \mathrm{M}$ & $1.0 \mathrm{M}$ & 7.9 & 6.7 & 0.0 & & & $x$ & & & & & $x$ & Adaptive Differential PCM decode \\
\hline \begin{tabular}{l|l}
$\mathrm{li}$ &
\end{tabular} & SpecInt95 & test & $1,340.0 \mathrm{M}$ & $1,151.7 \mathrm{M}$ & 5.5 & 2.8 & 2.7 & $\bar{x}$ & $\bar{x}$ & $\bar{x}$ & $\bar{x}$ & & & $\bar{x}$ & $\times$ & Lisp interpreter \\
\hline
\end{tabular}

Table 2: Benchmark Statistics and Characterization. All numbers are for the default SCALE configuration with four lanes. Results for multiple input data sets are shown separately if there was significant variation, otherwise an all data set indicates results were similar across inputs. As is standard practice, EEMBC statistics are for the kernel only. Total cycle numbers for non-EEMBC benchmarks are for the entire application, while the remaining statistics are for the kernel of the benchmark only (the kernel excludes benchmark overhead code and for $l i$ the kernel consists of the garbage collector only). The Mem B/Cycle column shows the DRAM bandwidth in bytes per cycle. The Loop Type column indicates the categories of loops which were parallelized when mapping the benchmark to SCALE: [DP] data-parallel loop with no control flow, [DC] data-parallel loop with conditional thread-fetches, [XI] loop with cross-iteration dependencies, [DI] data-parallel loop with inner-loop, [DE] loop with data-dependent exit condition, and [FT] free-running threads. The Mem column indicates the types of memory accesses performed: [VM] for vector-memory accesses and [VP] for individual VP loads and stores.

\begin{tabular}{|l||r|r|r|r|r|r|r|r|r|r|r|r|r|r|r|r|r|r|r|r|r|r|r|r|r|r|r|r|}
\hline & rgbcmy rgbyiq & hpg & text & dither & rotate & lookup & ospf & pktflw & pntrch & fir fbital & fft & viterb & autcor & conven & rijnd & sha|qsort & adpcm.e & adpcm.d & li.gc | & avg. \\
\hline \hline VP config: private regs & 2.0 & 1.0 & 5.0 & 2.7 & 10.0 & 16.0 & 8.0 & 1.0 & 5.0 & 7.0 & 4.0 & 3.0 & 9.0 & 3.6 & 3.0 & 6.0 & 13.0 & 1.0 & 26.0 & 4.0 & 1.0 & 4.4 & 6.2 \\
\hline VP config: shared regs & 10.0 & 18.0 & 3.0 & 3.6 & 16.0 & 3.0 & 9.0 & 5.0 & 12.0 & 14.5 & 2.0 & 8.0 & 1.0 & 3.9 & 2.0 & 7.0 & 5.0 & 3.8 & 20.0 & 19.0 & 17.0 & 5.1 & 8.5 \\
\hline vlmax & 52.0 & 120.0 & 60.0 & 90.8 & 28.1 & 24.0 & 40.0 & 108.0 & 56.0 & 40.0 & 64.0 & 116.0 & 36.0 & 49.8 & 124.0 & 40.0 & 28.0 & 113.5 & 12.0 & 48.0 & 96.0 & 112.7 & 66.3 \\
\hline vl & 52.0 & 120.0 & 53.0 & 6.7 & 24.4 & 18.5 & 40.0 & 1.0 & 52.2 & 12.0 & 60.0 & 100.0 & 25.6 & 16.6 & 32.0 & 31.7 & 4.0 & 5.5 & 12.0 & 47.6 & 90.9 & 62.7 & 39.5 \\
\hline
\end{tabular}

Table 3: VP configuration and vector-length statistics as averages of data recorded at each vector-fetch command. The VP configuration register counts represent totals across all four clusters, vlmax indicates the average maximum vector length, and $\mathrm{v} l$ indicates the average vector length.

\section{Loops with Cross-Iteration Dependencies}

Many loops are non-vectorizable because they contain loop-carried data dependencies from one iteration to the next. Nevertheless, there may be ample loop parallelism available when there are operations in the loop which are not on the critical path of the crossiteration dependency. The vector-thread architecture allows the parallelism to be exposed by making the cross-iteration (crossVP) data transfers explicit. In contrast to software pipelining for a VLIW architecture, the vector-thread code need only schedule instructions locally in one loop iteration. As the code executes on a vector-thread machine, the dependencies between iterations resolve dynamically and the performance automatically adapts to the software critical path and the available hardware resources.

Mediabench ADPCM contains one such loop (similar to Figure 8) with two loop-carried dependencies that can propagate in parallel. The loop is mapped to a single SCALE AIB with $35 \mathrm{VP}$ instructions. Cross-iteration dependencies limit the initiation inter- val to 5 cycles, yielding a maximum SCALE IPC of $35 / 5=7$. SCALE sustains an average of 6.7 compute-ops per cycle and achieves a speedup of $7.9 \times$ compared to the control processor.

The two MiBench cryptographic kernels, sha and rijndael, have many loop-carried dependences. The sha mapping uses 5 cross-VP data transfers, while the rijndael mapping vectorizes a short four-iteration inner loop. SCALE is able to exploit instruction-level parallelism within each iteration of these kernels by using multiple clusters, but, as shown in Figure 14, performance also improves as more lanes are added.

\section{Data-Parallel Loops with Inner-Loops}

Often an inner loop has little or no available parallelism, but the outer loop iterations can run concurrently. For example, the EEMBC lookup code models a router using a Patricia Trie to perform IP Route Lookup. The benchmark searches the trie for each IP address in an input vector, with each lookup chasing pointers through around 5-12 nodes of the trie. Very little parallelism is 

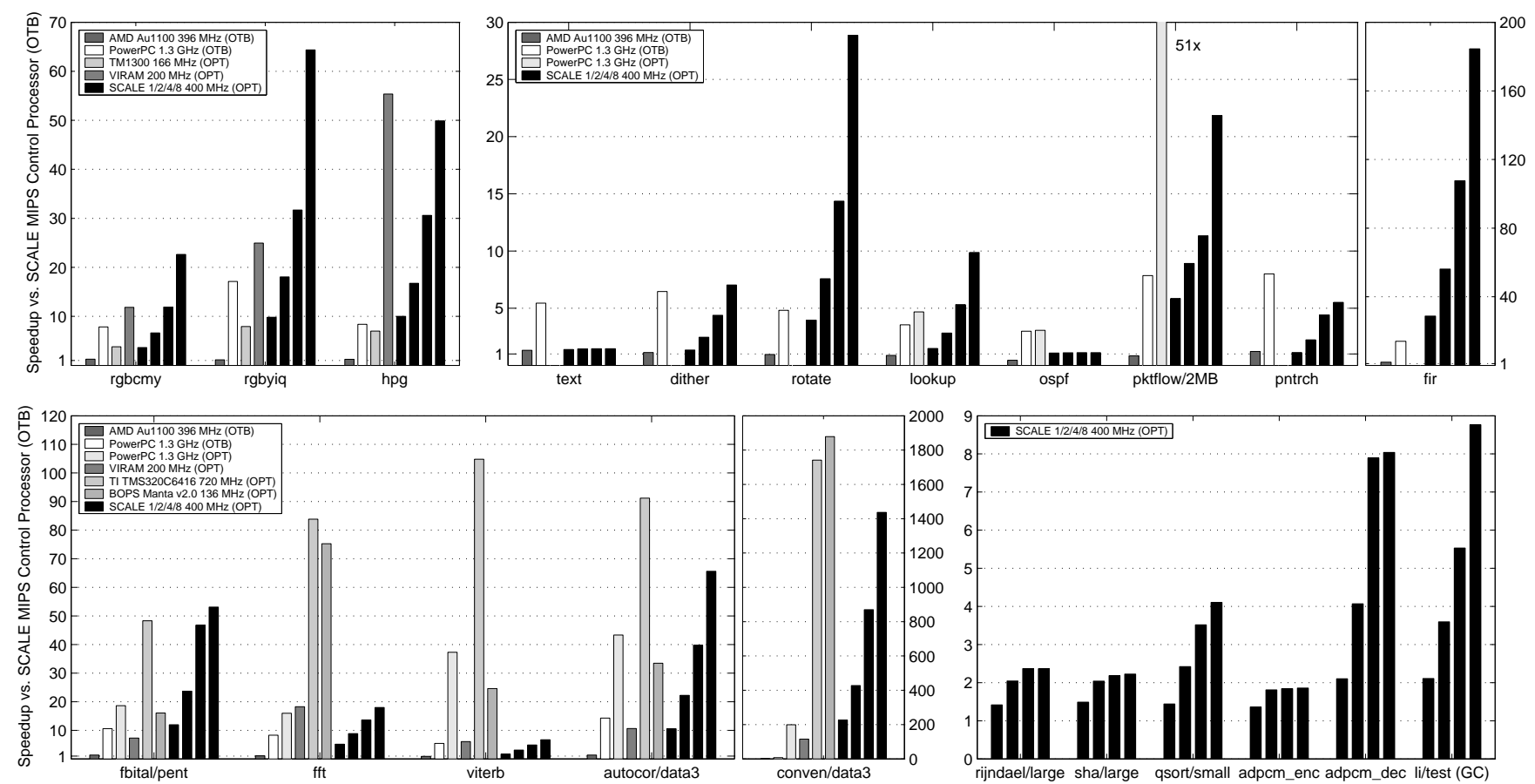

Figure 14: Performance Results: Twenty-two benchmarks illustrate the performance of four SCALE configurations $(1$ Lane, 2 Lanes, 4 Lanes, 8 Lanes) compared to various industry architectures. Speedup is relative to the SCALE MIPS control processor. The EEMBC benchmarks are compared in terms of iterations per second, while the non-EEMBC benchmarks are compared in terms of cycles to complete the benchmark kernel. These numbers correspond to the Kernel Speedup column in Table 2. For benchmarks with multiple input data sets, results for a single representative data set are shown with the data set name indicated after a forward slash.

available in each lookup, but many lookups can run simultaneously.

In the SCALE implementation, each VP handles one IP lookup using thread-fetches to traverse the trie. The ample thread parallelism keeps the lanes busy executing 6.3 ops/cycle by interleaving the execution of multiple VPs to hide memory latency. Vectorfetches provide an advantage over a pure multithreaded machine by efficiently distributing work to the VPs, avoiding contention for a shared work queue. Additionally, vector-load commands optimize the loading of IP addresses before the VP threads are launched.

\section{Reductions and Data-Dependent Loop Exit Conditions}

SCALE provides efficient support for arbitrary reduction operations by using shared registers to accumulate partial reduction results from multiple VPs on each lane. The shared registers are then combined across all lanes at the end of the loop using the cross-VP network. The pktflow code uses reductions to count the number of packets processed.

Loops with data-dependent exit conditions ("while" loops) are difficult to parallelize because the number of iterations is not known in advance. For example, the strcmp and strcpy standard C library routines used in the text benchmark loop until the string termination character is seen. The cross-VP network can be used to communicate exit status across VPs but this serializes execution. Alternatively, iterations can be executed speculatively in parallel and then nullified after the correct exit iteration is determined. The check to find the exit condition is coded as a cross-iteration reduction operation. The text benchmark executes most of its code on the control processor, but uses this technique for the string routines to attain a $1.5 \times$ overall speedup.

\section{Free-Running Threads}

When structured loop parallelism is not available, VPs can be used to exploit arbitrary thread parallelism. With free-running threads, the control processor interaction is eliminated. Each VP thread runs in a continuous loop getting tasks from a work-queue accessed using atomic memory operations. An advantage of this method is that it achieves good load-balancing between the VPs and can keep the VTU constantly utilized.

Three benchmarks were mapped with free-running threads. The pnt rch benchmark searches for tokens in a doubly-linked list, and allows up to five searches to execute in parallel. The qsort benchmark uses quick-sort to alphabetize a list of words. The SCALE mapping recursively divides the input set and assigns VP threads to sort partitions, using VP function calls to implement the compare routine. The benchmark achieves 2.3 ops/cycle despite a high cache miss rate. The ospf benchmark has little available parallelism and the SCALE implementation maps the code to a single VP to exploit ILP for a small speedup.

\section{Mixed Parallelism}

Some codes exploit a mixture of parallelism types to accelerate performance and improve efficiency. The garbage collection portion of the lisp interpreter ( $l i$ ) is split into two phases: mark, which traverses a tree of currently live lisp nodes and sets a flag bit in every visited node, and sweep, which scans through the array of nodes and returns a linked list containing all of the unmarked nodes. During mark, the SCALE code sets up a queue of nodes to be processed and uses a stripmine loop to examine the nodes, mark them, and enqueue their children. In the sweep phase, VPs are assigned segments of the allocation array and then each construct a list of unmarked nodes within their segment in parallel. Once the VP threads terminate, the control processor vector-fetches an AIB that stitches the individual lists together using cross-VP data transfers, thus producing the intended structure. Although the garbage collector has a high cache miss rate, the high degree of parallelism exposed in this way allows SCALE to sustain 2.8 operations/cycle and attain a 


\begin{tabular}{|c|c|c|c|c|c|c|c|c|c|c|c|c|c|c|c|c|c|c|c|c|c|c|}
\hline & rgbemy & rgbyiq & $\mathrm{hpg} g \mathrm{t}$ & text d & lither|r & rotate 1 & lookup & ospf] & pktflw & pntrch| & & fbital & $\mathrm{fft} \mid \mathrm{s}$ & viterb a & autcorpc & convent & rijnd] & sha| & qsort]a & pcm.ela & lpcm.d] & li.gc \\
\hline compute-ops / AIB & 21.0 & 29.0 & 3.7 & 4 & 8.6 & 19.7 & "5.11 & \begin{tabular}{c|c|}
16.5 \\
\end{tabular} & (4.2 & $7.0 \mid$ & $=4.0$ & $=7.4$ & 3.0 & 8.8 & 3.0 & $\overline{c 7.3}$ & $|13.4| 1$ & 14.1 & 9.1 & (61.0| & 35.0 & 8.9 \\
\hline compute-ops / AIB tag-check & 273.0 & 870.0 & 48.6 & 8.2 & 10.4 & 91.1 & 5.3 & 18.5 & 21.5 & 7.05 & 59.6 & 14.21 & 19.4 & 36.8 & 24.0 & 57.5 & 13.42 & 25.4 & 9.1 & 726.2 & $\begin{array}{l}795.3 \\
\end{array}$ & 12.2 \\
\hline compute-ops / ctrl. proc. instr. & 136.0 & 431.9 & \begin{tabular}{|c|}
44.7 \\
\end{tabular} & 0.2 & 23.7 & 85.7 & 639.2 & 857.8 & $152.3 \mid 3$ & $3189.6 \mid 1$ & 18.1 & \begin{tabular}{|l|}
62.8 \\
\end{tabular} & 5.8 & 4.9 & 23.6 & 19.7 & \begin{tabular}{|c|}
8.9 \\
\end{tabular} & 3.9 & 5.8 & 229.2 & 186.01 & 122.7 \\
\hline thread-fetches / VP thread & 0.0 & 0.0 & \begin{tabular}{|l|l|}
0.0 \\
\end{tabular} & 0.0 & 3.8 & 0.0 & 26.7 & 3969.0 & \begin{tabular}{|r|r|}
0.23 \\
\end{tabular} & \begin{tabular}{l|l|l|}
3023.7 & -1 \\
\end{tabular} & 0.0 & \begin{tabular}{|c|}
1.0 \\
\end{tabular} & 0.0 & 0.0 & 0.0 & 0.0 & 0.9 & \begin{tabular}{l|l}
0.0 & 1 \\
\end{tabular} & \begin{tabular}{|l|}
113597.9 \\
\end{tabular} & 0.0 & 0.0 & 2.4 \\
\hline AIB cache miss percent & 0.0 & 0.0 & 0.0 & 0.0 & 0.0 & 33.2 & 0.0 & 22.5 & 0.0 & 1.5 & 1.6 & 0.0 & 0.2 & 0.0 & 0.0 & 0.0 & 0.0 & 0.0 & $\begin{aligned} 4.3 \\
\end{aligned}$ & 0.0 & 0.1 & 0.4 \\
\hline
\end{tabular}

Table 4: Control hierarchy statistics. The first three rows show are the average number of compute-ops per executed AIB, per AIB tag-check (caused by either a vector-fetch, VP-fetch, or thread-fetch), and per executed control processor instruction. The next row shows the average number thread-fetches issued by each dynamic VP thread (launched by a vector-fetch or VP-fetch). The last row shows the miss rate for AIB tag-checks.

\begin{tabular}{|c|c|c|c|c|c|c|c|c|c|c|c|c|c|c|c|c|c|c|c|c|c|c|}
\hline & & & & & & & & & & & & & & & & & & & & & & \\
\hline sources: cl & 75.6 & 92.9 & 40.0 & 31.2 & \begin{tabular}{l|l|}
41.3 \\
\end{tabular} & 5.8 & 21.0 & 13.1 & 62.7 & 31.0 & 38.8 & 30.5 & 31.9 & 37.1 & 48.4 & 46.8 & 81.5 & 115.8 & \begin{tabular}{|l|}
32.6 \\
\end{tabular} & 20.3 & \begin{tabular}{|l|l||}
34.1 & 39.4 \\
\end{tabular} & $\overline{4.2}$ \\
\hline ource & 99.3 & 86.0 & 106.7 & & 94.21 & 109.8 & 113.6 & 127.0 & 84.7 & 115.0 & 113.3 & $114.4 \mid 8$ & 84.5 & 87.3 & 96.9 & 90.6 & 72.1 & 27.91 & & & \begin{tabular}{|l|l||}
110.177 .6 \\
\end{tabular} & 4.8 \\
\hline unces: in & 28.4 & 31.0 & \begin{tabular}{|l|l|}
6.7 \\
\end{tabular} & 13.1 & 27.2 & 64.0 & 21.8 & \begin{tabular}{|l|}
52.9 \\
\end{tabular} & 45.7 & 38.8 & 2.6 & \begin{tabular}{|l|}
30.2 \\
\end{tabular} & \begin{tabular}{|l|}
7.5 \\
\end{tabular} & 13.9 & 0.0 & 50.0 & 23.1 & 38.9 & 66. & 35.4 & \begin{tabular}{|l|l|l|}
38.7 & 71.7 \\
\end{tabular} & 32.2 \\
\hline sts: & 56.7 & $\begin{array}{c}58.5 \\
\end{array}$ & 40.0 & 18.2 & 43.8 & 5.8 & 21.8 & 18.5 & \begin{tabular}{ll|}
77.9 \\
\end{tabular} & 38.5 & 38.8 & $\mid \overline{22.8}$ & 31.9 & 37.1 & $\overline{48.4}$ & 40.6 & \begin{tabular}{|l|l|}
81.1 \\
\end{tabular} & 84.5 & $\overline{32}$ & & \begin{tabular}{|l|l||}
24.8 & 39.2 \\
\end{tabular} & 39.8 \\
\hline sis. & 33.8 & 31.2 & 60.0 & 52.8 & 46.0 & 59.6 & 48.2 & 87.3 & 52.6 & 23.1 & 60.7 & 83.0 & 51.2 & 64.9 & 51. & 75.0 & 22.0 & 15.5 & 43. & & \begin{tabular}{l|l|}
44.2 & 26.8 \\
\end{tabular} & 0.6 \\
\hline xt. cluster trans & 52.0 & 51.6 & 53.3 & 43.0 & \begin{tabular}{ll|}
45.9 \\
\end{tabular} & 34.5 & 36.6 & 53.5 & 57.6 & 30.9 & 38.8 & \begin{tabular}{|l|l|}
90.7 \\
\end{tabular} & 31.9 & 74.1 & 48.5 & 40 & 29.5 & 68.3 & 13.9 & 72.7 & $21.756 .3 \mid$ & 47.5 \\
\hline $\mathrm{ade}$ & $\overline{14.2}$ & 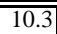 & 20.0 & 114.6 & "14.7 & $\overline{\overline{5.7}}$ & 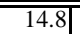 & |21.8 & $\overline{22.0}$ & \begin{tabular}{ll|}
15.4 \\
\end{tabular} & \begin{tabular}{c|}
19.0 \\
\end{tabular} & $\mid 15.12$ & 20.7 & \begin{tabular}{c|}
13.9 \\
\end{tabular} & $\overline{25.0}$ & 12.5 & $\mid 28.4$ & $\overline{\mid 15.4}$ & 18. & \begin{tabular}{|c|}
6.4 \\
\end{tabular} & $\begin{array}{l}9.3 \\
9.13 .0||\end{array}$ & 115.9 \\
\hline oad as & 14.2 & 10.3 & \begin{tabular}{l|}
5.3 \\
\end{tabular} & \begin{tabular}{|l|}
3.7 \\
\end{tabular} & 8.1 & 1.7 & 14.2 & 21.8 & 20.8 & 15.4 & 5.4 & \begin{tabular}{|l|}
9.4 \\
\end{tabular} & \begin{tabular}{|l|}
8.0 \\
\end{tabular} & 5.3 & 7. & 7.9 & 25.7 & 12.3 & & & \begin{tabular}{l|l||}
9.3 & 11.6 \\
\end{tabular} & 10.9 \\
\hline 10 & 14.2 & 10.3 & 20.0 & 14.6 & 30.2 & 5.7 & 59.1 & 87.4 & 54.2 & 61.6 & 75.9 & 30.2 & \begin{tabular}{|l|}
41.3 \\
\end{tabular} & 38.4 & 49.9 & 25.1 & \begin{tabular}{|l|}
113.4 \\
\end{tabular} & 61.7 & & 21.4 & 27.9 & 43.6 \\
\hline oad bytes from DRAM & 14.2 & 10.3 & 7.5 & \begin{tabular}{|l|}
0.0 \\
\end{tabular} & 2.9 & 0.3 & 0.2 & 0.0 & 115.3 & 0.0 & 0.4 & 0.0 & \begin{tabular}{|l|}
0.0 \\
\end{tabular} & 0.0 & 0.1 & 0.0 & \begin{tabular}{|l|}
0.0 \\
\end{tabular} & 0.0 & 59.1 & 0.0 & \begin{tabular}{ll|l|l|}
0.0 & 39.2 \\
\end{tabular} & 11.3 \\
\hline $\mathrm{ec}$ & $\overline{4 .}$. & & $\overline{6}$ & \begin{tabular}{c|c|}
4.8 \\
\end{tabular} & 3.5 & & & \begin{tabular}{ll|}
10.4 \\
\end{tabular} & $\overline{1.1}$ & $\overline{0 .}$. & & 0.5 & & & & & & & & & 9.5 & 5.2 \\
\hline $\mathrm{e}$ & 4. & 10 . & 1.8 & 1.4 & 1. & & 0. & 10.4 & 0. & 0. & 0.5 & 0.1 & 4.2 & 6 & & & 1 & & & & ग & \\
\hline ore bytes & 18.9 & 10.3 & 6.7 & 4.8 & 6.0 & 5.8 & 0.0 & 41.5 & 6.8 & 1.2 & 4.2 & 1.0 & 33.8 & 29.1 & 0.1 & 12.5 & 13.2 & 14.0 & 62. & & \begin{tabular}{ll||}
6.2 & 38.1 \\
\end{tabular} & 14.4 \\
\hline ore bytes to DRAM & 18.9 & 10.3 & 6.7 & \begin{tabular}{|l|}
0.5 \\
\end{tabular} & 0.7 & 0.0 & 0.0 & 0.0 & 8.4 & $\overline{0.0}$ & 0.1 & 0.0 & & 0.0 & & & & & 50.5 & $\overline{0.0}$ & & \\
\hline
\end{tabular}

Table 5: Data hierarchy statistics. The counts are scaled to reflect averages per 100 compute-ops executed in each benchmark, and the average (avg) column gives equal weight to all the benchmarks. Compute-op sources are broken down as coming from chain registers, the register file, or immediates; and compute-op and writeback-op destinations are broken down as targeting chain registers or the register file. The ext. cluster transports row reflects the number of results sent to external clusters. The load elements row reflects the number of elements accessed by either VP loads or vector-loads, while the load addresses row reflects the number of cache accesses. The load bytes row reflects the total number of bytes for the VP loads and vector-loads, while the load bytes from DRAM row reflects the DRAM bandwidth used to retrieve this data. The breakdown for stores corresponds to the breakdown for loads.

speedup of 5.5 over the control processor alone.

\subsection{Locality and Efficiency}

The strength of the SCALE VT architecture is its ability to capture a wide variety of parallelism in applications while using simple microarchitectural mechanisms that exploit locality in both control and data hierarchies.

A VT machine amortizes control overhead by exploiting the locality exposed by AIBs and vector-fetch commands, and by factoring out common control code to run on the control processor. A vector-fetch broadcasts an AIB address to all lanes and each lane performs a single tag-check to determine if the AIB is cached. On a hit, an execute directive is sent to the clusters which then retrieve the instructions within the AIB using a short (5-bit) index into the small AIB cache. The cost of each instruction fetch is on par with a register file read. On an AIB miss, a vector-fetch will broadcast AIBs to refill all lanes simultaneously. The vector-fetch ensures an AIB will be reused by each VP in a lane before any eviction is possible. When an AIB contains only a single instruction on a cluster, a vector-fetch will keep the ALU control lines fixed while each VP executes its operation, further reducing control energy.

As an example of amortizing control overhead, rbgy iq runs on SCALE with a vector-length of 120 and vector-fetches an AIB with 29 VP instructions. Thus, each vector-fetch executes 3,480 instructions on the VTU, 870 instructions per tag-check in each lane. This is an extreme example, but vector-fetches commonly execute 10s100 s of instructions per tag-check even for non-vectorizable loops such as adpcm (Table 4).

AIBs also help in the data hierarchy by allowing the use of chain registers, which reduces register file energy; and sharing of temporary registers, which reduces the register file size needed for a large number of VPs. Table 5 shows that chain registers comprise around $32 \%$ of all register sources and $44 \%$ of all register destinations. Table 3 shows that across all benchmarks, VP configurations use an average of 8.5 shared and 6.2 private registers, with an average maximum vector length above 64 (16 VPs per lane). The significant variability in register requirements for different kernels stresses the importance of allowing software to configure VPs with just enough of each register type.

Vector-memory commands enforce spatial locality by moving data between memory and the VP registers in groups. This improves performance and saves memory system energy by avoiding the additional arbitration, tag-checks, and bank conflicts that would occur if each VP requested elements individually. Table 5 shows the reduction in memory addresses from vector-memory commands. The maximum improvement is a factor of four, when each vector cache access loads or stores one element per lane. The VT architecture can exploit memory data-parallelism even in loops with non-data-parallel compute. For example, the fbital, text, and adpcm_enc benchmarks use vector-memory commands to access data for vector-fetched AIBs with cross-VP dependencies.

Table 5 shows that the SCALE data cache is effective at reducing DRAM bandwidth for most of the benchmarks. Two exceptions are the pktflow and $l i$ benchmarks for which the DRAM bytes transferred exceed the total bytes accessed. The current design always transfers 32-byte lines on misses, but support for nonallocating loads and stores could help reduce the bandwidth for these benchmarks.

Clustering in SCALE is area and energy efficient and cluster decoupling improves performance. The clusters each contain only a subset of all possible functional units and a small register file with few ports, reducing size and wiring energy. Each cluster executes compute-ops and inter-cluster transport operations in order, requiring only simple interlock logic with no inter-thread arbitra- 
tion or dynamic inter-cluster bypass detection. Independent control on each cluster enables decoupled cluster execution to hide large inter-cluster or memory latencies. This provides a very cheap form of SMT where each cluster can be executing code for different VPs on the same cycle (Figure 12).

\section{Related Work}

The VT architecture draws from earlier vector architectures [9], and like vector microprocessors [14, 6, 3] the SCALE VT implementation provides high throughput at low complexity. Similar to CODE [5], SCALE uses decoupled clusters to simplify chaining control and to reduce the cost of a large vector register file supporting many functional units. However, whereas CODE uses register renaming to hide clusters from software, SCALE reduces hardware complexity by exposing clustering and statically partitioning intercluster transport and writeback operations.

The Imagine [8] stream processor is similar to vector machines, with the main enhancement being the addition of stream load and store instructions that pack and unpack arrays of multi-field records stored in DRAM into multiple vector registers, one per field. In comparison, SCALE uses a conventional cache to enable unitstride transfers from DRAM, and provides segment vector-memory commands to transfer arrays of multi-field records between the cache and VP registers. Like SCALE, Imagine improves register file locality compared with traditional vector machines by executing all operations for one loop iteration before moving to the next. However, Imagine instructions use a low-level VLIW ISA that exposes machine details such as number of physical registers and lanes, whereas SCALE provides a higher-level abstraction based on VPs and AIBs.

VT enhances the traditional vector model to support loops with cross-iteration dependencies and arbitrary internal control flow. Chiueh's multi-threaded vectorization [1] extends a vector machine to handle loop-carried dependencies, but is limited to a single lane and requires the compiler to have detailed knowledge of all functional unit latencies. Jesshope's micro-threading [2] uses a vector-fetch to launch micro-threads which each execute one loop iteration, but whose execution is dynamically scheduled on a per-instruction basis. In contrast to VT's low-overhead direct cross-VP data transfers, cross-iteration synchronization is done using full/empty bits on shared global registers. Like VT, Multiscalar [12] statically determines loop-carried register dependencies and uses a ring to pass cross-iteration values. But Multiscalar uses speculative execution with dynamic checks for memory dependencies, while VT dispatches multiple non-speculative iterations simultaneously. Multiscalar can execute a wider range of loops in parallel, but VT can execute many common parallel loop types with much simpler logic and while using vector-memory operations.

Several other projects are developing processors capable of exploiting multiple forms of application parallelism. The Raw [13] project connects a tiled array of simple processors. In contrast to SCALE's direct inter-cluster data transfers and cluster decoupling, inter-tile communication on Raw is controlled by programmed switch processors and must be statically scheduled to tolerate latencies. The Smart Memories [7] project has developed an architecture with configurable processing tiles which support different types of parallelism, but it has different instruction sets for each type and requires a reconfiguration step to switch modes. The TRIPS processor [10] similarly must explicitly morph between instruction, thread, and data parallelism modes. These mode switches limit the ability to exploit multiple forms of parallelism at a fine-grain, in contrast to SCALE which seamlessly combines vector and threaded execution while also exploiting local instruction-level parallelism.

\section{Conclusion}

The vector-thread architectural paradigm allows software to more efficiently encode the parallelism and locality present in many applications, while the structure provided in the hardware-software interface enables high-performance implementations that are efficient in area and power. The VT architecture unifies support for all types of parallelism and this flexibility enables new ways of parallelizing codes, for example, by allowing vector-memory operations to feed directly into threaded code. The SCALE prototype demonstrates that the VT paradigm is well-suited to embedded applications, allowing a single relatively small design to provide competitive performance across a range of application domains. Although this paper has focused on applying VT to the embedded domain, we anticipate that the vector-thread model will be widely applicable in other domains including scientific computing, high-performance graphics processing, and machine learning.

\section{Acknowledgments}

This work was funded in part by DARPA PAC/C award F3060200-2-0562, NSF CAREER award CCR-0093354, an NSF graduate fellowship, donations from Infineon Corporation, and an equipment donation from Intel Corporation.

\section{References}

[1] T.-C. Chiueh. Multi-threaded vectorization. In ISCA-18, May 1991.

[2] C. R. Jesshope. Implementing an efficient vector instruction set in a chip multi-processor using micro-threaded pipelines. Australia Computer Science Communications, 23(4):80-88, 2001.

[3] K. Kitagawa, S. Tagaya, Y. Hagihara, and Y. Kanoh. A hardware overview of SX-6 and SX-7 supercomputer. NEC Research \& Development Journal, 44(1):2-7, Jan 2003.

[4] C. Kozyrakis. Scalable vector media-processors for embedded systems. PhD thesis, University of California at Berkeley, May 2002.

[5] C. Kozyrakis and D. Patterson. Overcoming the limitations of conventional vector processors. In ISCA-30, June 2003.

[6] C. Kozyrakis, S. Perissakis, D. Patterson, T. Anderson, K. Asanović, N. Cardwell, R. Fromm, J. Golbus, B. Gribstad, K. Keeton, R. Thomas, N. Treuhaft, and K. Yelick. Scalable Processors in the Billion-Transistor Era: IRAM. IEEE Computer, 30(9):75-78, Sept 1997.

[7] K. Mai, T. Paaske, N. Jayasena, R. Ho, W. Dally, and M. Horowitz. Smart Memories: A modular reconfigurable architecture. In Proc. ISCA 27, pages 161-171, June 2000.

[8] S. Rixner, W. Dally, U. Kapasi, B. Khailany, A. Lopez-Lagunas, P. Mattson, and J. Owens. A bandwidth-efficient architecture for media processing. In MICRO-31, Nov 1998.

[9] R. M. Russel. The CRAY-1 computer system. Communications of the ACM, 21(1):63-72, Jan 1978.

[10] K. Sankaralingam, R. Nagarajan, H. Liu, C. Kim, J. Huh, D. Burger, S. W. Keckler, and C. Moore. Exploiting ILP, TLP, and DLP with the polymorphous TRIPS architecture. In ISCA-30, June 2003.

[11] J. E. Smith. Dynamic instruction scheduling and the Astronautics ZS-1. IEEE Computer, 22(7):21-35, July 1989.

[12] G. S. Sohi, S. E. Breach, and T. N. Vijaykumar. Multiscalar processors. In ISCA-22, pages 414-425, June 1995.

[13] E. Waingold, M. Taylor, D. Srikrishna, V. Sarkar, W. Lee, V. Lee, J. Kim, M. Frank, P. Finch, R. Barua, J. Babb, S. Amarasinghe, and A. Agarwal. Baring it all to software: Raw machines. IEEE Computer, 30(9):86-93, Sept 1997.

[14] J. Wawrzynek, K. Asanović, B. Kingsbury, J. Beck, D. Johnson, and N. Morgan. Spert-II: A vector microprocessor system. IEEE Computer, 29(3):79-86, Mar 1996.

[15] M. Zhang and K. Asanović. Highly-associative caches for low-power processors. In Kool Chips Workshop, MICRO-33, Dec 2000. 